\title{
Diagnosis and Grading Of Papilledema Using Optical Coherence Tomography Compared To Clinical Staging By Frise'n Scale.
}

\author{
Manar T. Mohamed ${ }^{\mathrm{a}}$, Ahmed H.Aldghaimy ${ }^{\mathrm{a}}$,Osama A.Elsoghair ${ }^{\mathrm{a}}$,WaelElshazlyAita ${ }^{\mathrm{a}}$ \\ ${ }^{a}$ Ophthalmology Department, Qena Faculty of Medicine, South Valley University.
}

\section{Abstract:}

Background:Papilledema, or optic disc swelling due to raised intracranial pressure, has been graded using the Frise' n Scale. In this study we aim to compare and contrast 2 methods of quantitating papilledema, namely, Optical coherence tomography (OCT) and Modified Frise'n Scale (MFS).

Materials and method:This study was performed at ophthalmology department, Qena Faculty of Medicine, South Valley University from June 2019 to January 2020, thirty patients with papilledema were enrolled, assessment of OCT retinal nerve fiber layer thickness, OCT total retinal thickness, then compared by modified Frise'n scale from photographs.

Results:Our study included 30 patients, 26 females with mean age $28.2 \pm 6.7$ and 4 males with mean age $32 \pm 2$, Among 30 patients with papilledema: 7(21) \% had grade $1,10(30) \%$ had grade $2,4(12) \%$ had grade $3,9(27) \%$ had grade 4 . When OCT RNFL thickness was compared with MFS grade from photographs (using majority rule), Spearman rank correlation was 0.727 ( $\left.\mathrm{P}_{-} .0005\right)$. When OCT total retinal thickness was compared with MFS grade from photographs, Spearman rank correlation was 0.789(P_.0005).

Conclusions: In grading papilledema, we found strong correlation between the modified Frise'n scale and OCT findings. For lower-grade optic disc edema, OCT compares better with clinical staging of optic nerve head photographs. With higher grades of edema, OCT RNFL thickness processing algorithms often fail or lack sensitivity, while OCT total retinal thickness performing more favorably.

Keywords:Papilledema, Optical coherence tomography (OCT), modified Frise'n scale (MFS).

\section{Introduction}

Papilledema, or optic disc swelling due to raised intracranial pressure, has been graded using the Frise' n Scale. This scale uses visual features of the optic disc and peripapillary retina to stage optic disc edema. Its reproducibility has been validated (Frise'n., 1982)but it is limited by use of an ordinal scale.

Optical coherence tomography (OCT) is a potential tool to quantify changes in the degree of papilledema and to monitor the efficacy of treatment interventions. First described by (Huang et al., 1991).
OCT is a cross-sectional imaging technique that quantitatively assesses multiple layers of the retina, allowing measurement of the retinal nerve fiber layer (RNFL) with a resolution of approximately $10 \mu \mathrm{m}$ using time domain variables(Baumal., 1999., Schuman., 1995., Paunescu et al., 2004).Direct measurements are calculated by a computer algorithm to quantify the nerve fiber layer and total retinal thickness.

OCT offers several advantages over conventional photographic imaging such as use with small pupil sizes and nuclear 
cataracts. Despite these advantages, limitations of OCT include the requirement for patient fixation stability during imaging without eye tracking and the failure of segmentation algorithms for defining RNFL thickness and total retinal thickness in eyes with severe papilledema.

Although fundus photographs require subjective interpretation, they show detailed topographic relationships of opticdisc edema that may be difficult to quantifyand document on clinical examination.

Unlike OCT, phōographic artifacts (eg, defocus or incorrect light exposure) that could interfere with interpretation areusually obvious.

Few studies evaluated theuseofOCTinmeasuringRNFLor total retinalthickening due to papilledema. A 2007Study(El-Dairi et al., 2007) compared the findings of OCT versusdisc photographs in children with idiopathicintracranial hypertension. Anotherstudy(Uysal et al.,2006) assessed the importance of RNFLthickness analysis in intracranial hypertensionusing imaging laser polarimetry. (Hoye et al., 2001)used OCT to demonstrate subretinalmacular edema in papilledema.

OCT has been usedto measure RNFL thickness in patients with optic neuritis (Parisi et al., 2003). Other studies(Ophir, Karam, Rebolleda et al.,20052009)compared OCT (as well as automatedperimetry)RNFLthickness in papilledema and pseudopapilledemabut did not

compareOCTversusfundusphotograph grades. To our knowledge, this is the first studyto compare OCT RNFL thickness and OCT total retinalthickness with fundus disc photographs in adults havingraised intracranial pressure. Our objectives were to compareand contrast OCT and Modified Frise'n Scale (MFS)grading with the peripapillary RNFL in quantifying optic disc edema.

\section{MATERIALS \& METHOD}

- Study design: A prospective, cohort, hospital based study carried out on patientswith papilledema in ophthalmology Department in Qena university hospital from June 2019 to January 2020.

- Sample size: 30 patients of both sexes were examined from outpatient clinic and inpatient wards of ophthalmology department in Qena University hospital.

Target population:

a. Inclusion criteria:Adult patients with raised intracranial tension above 18 years old.

b. Exclusion criteria:Children and uncooperative patients with disturbed conscious level. No other cause of visual loss or optic disc edema was present such as optic nerve compression, inflammatory optic neuropathy, or ischemic optic neuropathy.

\section{Methodology}

All of patients had been subjected to the following:

-Full history and clinical examination.

-Visual field examination.

-Fundus photography.

-OCT measurement of retinal nerve fiber layer thickness and OCT total retinal thickness.

\section{- For OCT analysis:}

The circular image had to be centred on the nerve, the signal strength had to be at least 5 (range, 1[lowest]-10 [highest]), and the proprietary algorithm used by the manufacturer (OCT spectralis, Heidelberg Engineering) had to successfully delineate the borders of the 
RNFL onmanual inspection of the images. The proprietary algorithm creates a best fit estimate of the inner and outer borders of the RNFL and the total retinal thickness to calculate the mean thickness.

\section{- For Fundus photographs:}

The optic nerve had to be in focus to allow evaluation of the RNFL at the disc border. Analysis was monocular rather than binocular because of some poor photographic quality or failure to capture the entire extent of the z-axis by OCT due to peripapillary elevation and cropping of the B-scan in 1 of 2 eyes. Furthermore, use of both eyes would violate a principle of parametric statistics, as the results of one eye are not independent of the other eye. Although the relationship between eyes could be accounted for with nested analysis of variance, using both eyes in some patients would have complicated our statistical analysis.

\section{Table [1]: Modified Frise'n Scale(Frise'n., 1982)}

\begin{tabular}{|c|}
\hline $\begin{array}{l}\text { 0 (Normal Optic Disc) } \\
\text { nence of the retinal nerve fiber } \\
t \text { the nasal, superior, and } \\
\text { poles in inverse proportion to } \\
\text { ameter. } \\
\text { nerve fiber layer striations, } \\
\text { t tortuosity. }\end{array}$ \\
\hline $\begin{array}{l}1 \text { (Minimal Degree of Edema) } \\
\text {-C-shaped halo that is subtle and grayish } \\
\text { with a temporal gap; obscures } \\
\text { underlying retinal details. } \\
\text {-Disruption of normal radial nerve fiber } \\
\text { layer arrangement striations. } \\
\text {-Temporal disc margin normal. }\end{array}$ \\
\hline
\end{tabular}

\begin{tabular}{|l|}
\hline -Circumferential halo. \\
-Elevation (nasal border). \\
-No major vessel obscuration. \\
\hline \multicolumn{1}{|c|}{$\mathbf{3}$ (Moderate Degree of Edema) } \\
-Obscuration of _1 segment of major \\
blood vessels leaving disc. \\
-Circumferential halo. \\
-Elevation (all borders). \\
-Halo (irregular outer fringe with finger- \\
like extensions). \\
\hline \multicolumn{1}{|c|}{ (Marked Degree of Edema) } \\
-Total obscuration on the disc of a \\
segment of a major blood vessel on \\
the disc. \\
-Elevation (whole nerve head, including \\
the cup). \\
-Border obscuration (complete). \\
-Halo (complete). \\
\hline Grade 5 (Severe Degree of Edema) \\
-Obscuration of all vessels on the disc \\
and leaving the disc.
\end{tabular}

\section{Statistical analysis:}

All analyses were performed with the IBM SPSS 20.0 software. The data were tested for normality using the Anderson Darling test and for homogeneity variances prior to further statistical analysis. Categorical variables were described by number and percent (N \& \%), where continuous variables described by mean and standard deviation (Mean, SD). Chi-square used for comparison between categorical variables, whereas independent samples t-test and paired t-test used to compare between continuous variables.

\section{Results}

\section{Demographic data:}

30 patients with papilledema,26 females with mean age $28.2 \pm 6.7$ and 4 males with mean age $32 \pm 2$.

\section{correlation between the MFS and OCT findings:}

In our study we found that $7(21) \%$ had grade $1,10(30) \%$ had grade $2,4(12) \%$ had grade 3, 9(27) \% had grade 4 .

OCT RNFL thickness and total retinal thickness showed significant correlation 
with the MFS grade from photographs(table 1) In grading papilledema, we found strong correlation between the MFS and OCT findings. When OCT RNFL thickness was compared with MFS grade from photographs (using majority rule), Spearman rank correlation was 0.727 ( $\left.\mathrm{P}_{-} .0005\right)$ in figure 1 . When OCT total retinal thickness was compared with MFS grade from photographs, Spearman rank correlation was $0.789\left(\mathrm{P}_{-} .0005\right)$ in figure 2 .

Table [2]: Correlation between total retinal thickness, nerve fiber layer thickness and modified Frise'n scale grading:

\begin{tabular}{|ll|r|}
\hline & & \multicolumn{1}{|c|}{ GRADE } \\
\hline Total & Pearson & $.789^{* * *}$ \\
retinal & Correlation & \\
thichness & Sig. (2-tailed) & .0005 \\
& $\mathrm{~N}$ & 30 \\
\hline RNFL & Pearson & $.727^{* * *}$ \\
thickness & Correlation & \\
& Sig. (2-tailed) & .0005 \\
& $\mathrm{~N}$ & 30 \\
\hline
\end{tabular}

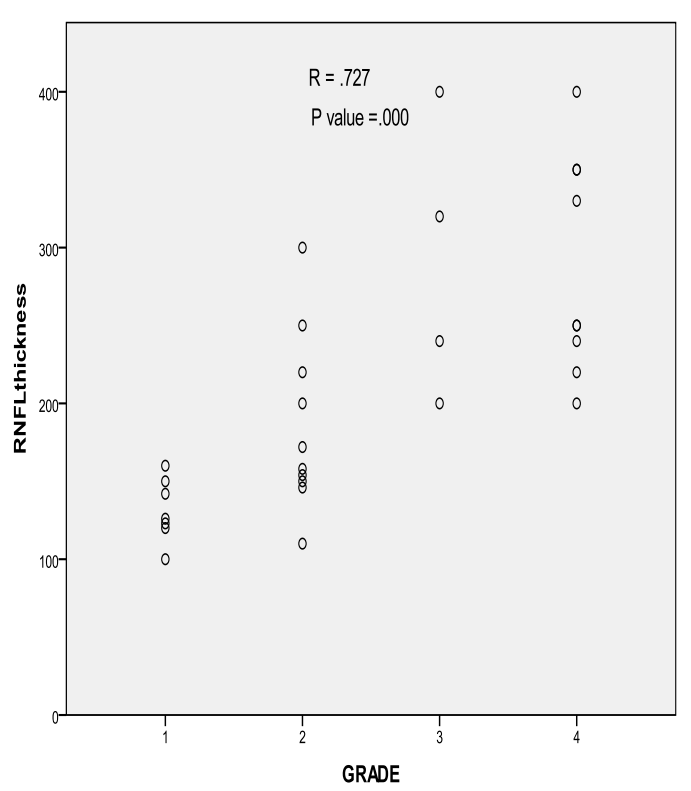

Figure (1): Papilledema grading differences in 30 patients using optical coherence tomography (OCT) retinal nerve fiber layer (RNFL) thickness versus using Modified Frise'n Scale.

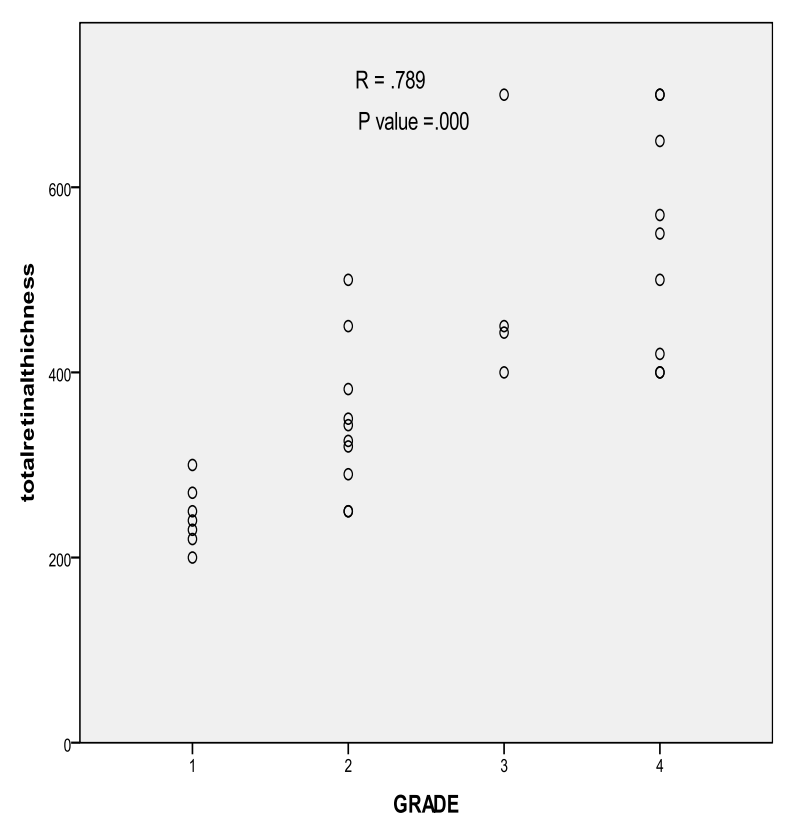

Figure (2): Papilledema grading differences in 30 patients using OCT total retinal thickness vs using Modified Frise'nScale grade.

\section{Discussion}

In the current study, we found strong Spearman rank correlation between OCT 
RNFL thickness, OCT total retinal thickness, and MFS grade from photographs.

In grading papilledema, we found strong correlation between the MFS and OCT findings. When OCT RNFL thickness was compared with MFS grade from photographs (using majority rule), Spearman rank correlation was 0.727 $\left(P_{.} .0005\right)$. When OCT total retinal thickness was compared with MFS grade from photographs, Spearman rank correlation was 0.789 (P_.0005).

The effect of papilledema can be seen on RNFL thickness and on total retinal thickness. A disproportional increase of total retinal thickness above that of the RNFL represents fluid from neurosensory retinal detachment in the peripapillary retina. Therefore, OCT total retinal thickness may show proportionally. axonal transport materials in the RNFL in optic disc edema could increase peripapillary RNFL reflectance and induce than representing it. (Johnson et al., )found RNFL thickness in the nasal section to be a good differential marker for optic disc edema from ONHD, with a thinner cut-off value of 78 microns (Lee et al., 2011).

The correlation between OCT and fundus appearance of the disc was greatest when photographs were ranked in continuous order of edema severity. Variation in OCT measurements at each grade could be partly explained by the ordinal character of the MFS, as ranking the photographs in order within each grade resulted in higher correlation between OCT findings and MFS grade.

OCT can objectively quantify the amount of RNFL swelling, and thus axonal swelling, which is the main pathological event in optic nerve edema;(Tsoet al.,1977),(Savini et al.,2006).

Fundus photographs will remain an important and reliable method for the assessment of disc edema because of their availability, use among multiple medical fields, and few but obvious artifacts, as well as the ability of the examiner to individually assess edema using multiple keys.

\section{Conclusion:}

OCT total retinal thickness may show proportionally more change per degree of disc edema than OCT RNFL thickness. More increase of total retinal thickness above that of the RNFL represents fluid from neurosensory retinal detachment in the peripapillary retina. For lower-grade optic disc edema, OCT compares better with clinical staging of optic nerve head photographs. With higher grades of edema, OCT RNFL thickness processing algorithms often fail or lack sensitivity, while OCT total retinal thickness performing more favorably.

\section{Study Limitations:}

We have several limitations in our study;our sample size is small, especially at the higher papilledema grades. Measurement error can occur with OCT and with assessment of fundus photographs, and OCT algorithm failure rates can become significant with high grades of papilledema .Disc hemorrhages can also affect accuracy, as they may influence reflectivity in an inconsistent manner.

\section{References}

Frise'n L, (1982).Swelling of the optic nerve head: a staging scheme. J NeurolNeurosurgPsychiatry. $\quad 45(1)$ :1318.

Huang D, Swanson EA, Lin CP, Schuman JS, Stinson WG, Chang W, et al, (1991).Optical coherence tomography. Science.254(5035):11781181. 
Baumal CR, (1999). Clinical applications of optical coherence tomography. CurrOpinOphthalmol. 10(3):182-188.

Schuman JS, Hee MR, Puliafito CA, Wong C, Lin CP, Pedut-Koizman, et al, (1995).Quantification of nerve fiber layer thickness in normal and glaucomatous eyes using optical coherence tomography. Arch Ophthalmol. 113(5):586-596.

Paunescu LA, Schuman JS, Price LL, Lelia A, Joel S, (2004).Reproducibility of nerve fiber thickness, macular thickness, and optic nerve head measurements using Stratus OCT. Invest Ophthalmol Vis Sci. 45(6):1716-1724.

El-Dairi MA, Holgado S, O'Donnell T, Buckley EG, Asrani S, Freedman SF, (2007).Opticalcoherence tomography as a tool for monitoring pediatric pseudotumorcerebri. JAAPOS. 11(6):564-570.

Uysal TF, Cengiz A, Reyhan G, Hatice D, (2006). Retinal nerve fiber layer analysis in idiopathicintracranial hypertension. NeurolIndia.54(2):168172.

Hoye VJ III, Berrocal AM, Hedges TR III,Amaro-Quireza ML, (2001).Optical coherencetomography demonstrates subretinal macular edema from papilledema. ArchOphthalmol. 119(9):1287-1290.

Parisi V, Manni G, Spadaro M,Colacino G, Restuccia R, Marchi S, et al, (1999).Correlation between morphological and functionalretinal impairment in multiple sclerosis patients. Invest OphthalmolVisSci.40(11):25202527.

Parisi V, (2003).Correlation between morphological and functional retinalimpairmentin patients affected by ocular hypertension, glaucoma, demyelinating optic neuritisand Alzheimer's disease. SeminOphthalmol. 18(2):50-57.

Ophir A, Karatas M, Ramirez JA, InzelbergR, (2005).OCT and chronic papilledema.Ophthalmology. 112(12):2238.

Karam EZ, Hedges TR, (2005).Optical coherence tomography of the retinal nerve fibrelayer in mild papilloedema and pseudopapilloedema. $\mathrm{Br} \mathbf{J}$ Ophthalmol. 89(3):294-298.

Rebolleda G, Muñoz-Negrete FJ, (2009).Follow-up of mild papilledema in idiopathic intracranialhypertension with optical coherence tomography. InvestOphthalmolVis Sci. 50(11):51975200.

Lee KM, Woo SJ, Hwang JM, (2011).Differentiation of optic nerve head drusen and optic disc edema with spectral domain optical coherence tomography.Ophthalmology.118:971-7.

Tso MO, Hayreh SS, (1977).Optic disc edema in raised intracranial pressure III: a pathologic study of experimental papilledema. Arch Ophthalmol. 95:1448-57.

Savini G, Bellusci C, Carbonelli M, Zanini M, Carelli V, Sadun AA, et al, (2006). Detection and quantification of retinal nerve fiber layer thickness in optic disc edema using stratus OCT. Arch Ophthalmol. 124:1111-7. 